\title{
Simultaneous HPLC Determination of Efavirenz, 8-Hydroxy Efavirenz, Neostigmine and Comparison of Separation Using a C18 and Biphenyl Column through Pharmacological Evaluation
}

\author{
S. KUMAR*, P. J. BOUIC ${ }^{1,2}$ AND B. ROSENKRANZ
}

Division of Clinical Pharmacology, Department of Medicine, University of Stellenbosch, Cape Town, 7505, ${ }^{1 S y n e x a}$ Life Sciences, Montague Gardens, Cape Town, 7441, ${ }^{2}$ Division of Medical Microbiology, Department of Medicine, University of Stellenbosch, Cape Town, 7505, South Africa

Kumar, et al.: HPLC Determination of Efavirenz, 8-Hydroxy Efavirenz and Neostigmine

\begin{abstract}
A simple, rapid and stable high performance liquid chromatography method for a combination of efavirenz, its major metabolite 8-hydroxy efavirenz and neostigmine was developed and validated. The drugs individually and in combination, were analysed using an Agilent 1260 high performance liquid chromatography coupled with variable wavelength detector. Successful separation of combined drugs were achieved by gradient elution on a reverse-phase C18 Phenomenex Evo $100 \mathrm{~A}$ column $(150 \times 4.6 \mathrm{~mm}, 2.6 \mu)$, by gradient elution using a mobile phase consisting of water:acetonitrile at $0.6 \mathrm{ml} \times \mathrm{min}^{-1}$ flow rate, detection wavelength $245 \mathrm{~nm}$, column oven temperature $27^{\circ}$ and injection volume $15 \mu$ l. The same method was also deployed on a Restek Ultra biphenyl column $(150 \times 4.6 \mathrm{~mm}, 5 \mu)$ to compare the levels of separation of the drugs and the metabolite. The chromatographic retention times were consistent at 8.75, 9.33 and 10.20 min for efavirenz, 8-hydroxy efavirenz and neostigmine, respectively. Polynomial regression data for the calibration plots exhibited linear relationship (correlation coefficient $=0.999$ ) in the range of $2.5-150 \mu \mathrm{M}$ for both efavirenz and 8-hydroxy efavirenz, and the lower limit of detection and lower limit of quantification at 32 and $96.97 \mu \mathrm{M}$ for efavirenz and 29.49 and $89.38 \mu \mathrm{M}$ for 8-hydroxy efavirenz, respectively. Evaluation of drug metabolism using human liver microsomes could be achieved with this method and linearity was established for the 30,45 and 60 min incubations (correlation coefficient $=0.97$ ). The method could be a potential tool to investigate herb and drugdrug interactions as well as for quantifying drugs in in vitro drug metabolism assays.
\end{abstract}

Key words: HPLC, C18, efavirenz, 8-hydroxy efavirenz, neostigmine, biphenyl column, human liver microsomes

Efavirenz $\left(\mathrm{EFV}, \mathrm{C}_{14} \mathrm{H}_{9} \mathrm{ClF}_{3} \mathrm{NO}_{2}\right)$ is an antiretroviral (ARV) agent employed in the treatment of human immunodeficiency virus (HIV) type 1 in combination with other drugs. It is an analogue of non-nucleoside ARV agent and a non-competitive reverse transcriptase inhibitor and is directly connected to the enzyme and blocks RNA and DNA-dependent, DNA-polymerase activities, causing destruction of the enzyme catalytic site. Chemicallyit is(S)-6-chloro-(cyclopropylethynyl)1,4-dihydro-4-(trifluoromethyl)-2H-3,1-benzoxazin2-one and is optically active with a molecular weight of $315.68 \mathrm{~g} / \mathrm{mol}$ and melting point in the range 139 $141^{\circ}$. This crystalline powder has a white or slightly yellowish appearance. The substance has a melting point ranging from $136-141^{\circ}$, is nearly water insoluble but soluble in methanol and dichloromethane ${ }^{[1]}$. EFV

*Address for correspondence E-mail: saneesh.7.kumar@gmail.com

May-June 2017 is predominantly cleared by hepatic metabolism ${ }^{[2]}$. The metabolites identified in human plasma and urine (almost exclusively as glucuronide or sulphate conjugates) were 7- and 8-hydroxy efavirenz (primary metabolites) and 8,14-dihydroxy efavirenz (secondary metabolite). 8-hydroxy efavirenz (E8H, $\mathrm{Cl}_{4} \mathrm{H}_{9} \mathrm{ClF}_{3} \mathrm{NO}_{3}$ ) is the major metabolite from EFV primarily formed via CYP2B6 of the cytochrome $\mathrm{P}_{450}$ system $^{[3]}$. It has the IUPAC name (4S)-6-chloro-4-(2-

This is an open access article distributed under the terms of the Creative Commons Attribution-NonCommercial-ShareAlike 3.0 License, which allows others to remix, tweak, and build upon the work non-commercially, as long as the author is credited and the new creations are licensed under the identical terms

Accepted 06 April 2017

Revised 31 December 2016

Received 13 September 2016

Indian J Pharm Sci 2017;79(3):353-360 
cyclopropylethynyl)-7-hydroxy-4-(trifluoromethyl)1H-3,1-benzoxazin-2-one with molecular weight $331.675 \mathrm{~g} / \mathrm{mol}$ and melting point in the range of 186$188^{\circ}$. Several analytical methods have been previously reported for the determination of EFV in human plasma, using either high-performance liquid chromatography using ultra-violet detection (HPLC-UV) ${ }^{[4-6]}$ or liquid chromatography with mass spectrometric (LC-MS) detection $^{[2,7]}$. HPLC-UV is, owing to its lower cost and greater robustness, still widely used for relatively less complex bioanalytical assays ${ }^{[8]}$. Also HPLC methods have already been developed to detect and separate EFV and its major metabolites ${ }^{[3]}$. This is of critical importance especially in assays involving human liver microsomes (HLM), to identify the metabolites and also quantify the rate of drug metabolism. Metabolites may interfere when analysing parent drugs ${ }^{[9]}$. It is important that potential interferences from all these compounds are part of the method. Drugs entering the body undergo biotransformation via phase I and phase II metabolic pathways. Based on the nature of the chemical reactions involved, metabolites formed from phase I reactions are more likely to be chemically reactive or pharmacologically active and therefore, more likely to need safety evaluation ${ }^{[10]}$.

Neostigmine methyl sulphate $\left(\mathrm{NEO}, \mathrm{C}_{13} \mathrm{H}_{22} \mathrm{~N}_{2} \mathrm{O}_{6} \mathrm{~S}\right)$ with molecular weight $334.387 \mathrm{~g} / \mathrm{mol}$, IUPAC name [3-(dimethylcarbamoyloxy)phenyl]-trimethylazanium; methyl sulphate and melting point in the range of 145$149^{\circ}$, is a cholinesterase inhibitor used in the treatment of myasthenia gravis and to reverse the effects of muscle relaxants such as gallamine and tubocurarine. Studies have shown that, just as in myasthenia gravis, most of the lymphocytic infiltrate in HIV-1 infected thymuses is located within the perivascular space surrounding the thymic epithelium and contains peripheral CD8 cytotoxic $\mathrm{T}$ lymphocyte (CTL) effector cells ${ }^{[11]}$. The co-administration of neostigmine and ARVs in such cases has proven to be more effective ${ }^{[12]}$.

The present study describes an HPLC-variable wavelength detector (VWD) method for the detection, separation and quantitation of EFV and E8H standard drugs, that is rapid with a shorter analysis time and selective in the presence of not only first-line ARV but also reversible acetylcholinesterase inhibitor drugs such as NEO as an internal standard. Also, a comparison of the separation methodology is achieved using a C18 column and a biphenyl column, since biphenyl columns have the capability to give better separation for compounds with ring structure. The method specificity is validated using in vitro HLM assays to analyse the robustness of the method to separate the drug, metabolite and the internal standard in exact consistency as the standards, in a complex matrix comprising of other sub-metabolites/cofactors facilitating the metabolism. The method is also unique in the sense it deploys a reverse phase gradient involving normal HPLC solvents and no buffers, unlike the other HPLC methods available today. The separation of the drug, its metabolite and the internal standard is satisfactorily stable and there is minimal interference from other sub-metabolites and cofactors involved in the actual HLM drug metabolism assays.

\section{MATERIALS AND METHODS}

Pure E8H and NEO were obtained from Clearsynth Labs Ltd. (Mumbai, India) while EFV was obtained from Sigma-Aldrich (Steinheim, Germany). HPLCgrade acetonitrile and methanol was purchased from Sigma-Aldrich (Steinheim, Germany). High purity water was prepared by passage through a Millipore system (Millipore, Billerica, MA, USA) and was used as the mobile phase. The HLM screening was performed using pooled HLM (mixed gender) from Xenotech LLC, USA along with glucose-6-phosphate sodium salt, glucose-6-phosphate dehydrogenase, magnesium chloride, phosphate buffer solution $1 \mathrm{M}$ and $\beta$-nicotinamide adenine dinucleotide phosphate hydrate (NADPH) from Sigma-Aldrich (Steinheim, Germany).

All stock solutions for the drugs were prepared in 4 $\mathrm{mM}$ concentrations, in methanol and the dilutions were made from the same with diluents: a mixture of mobile phase water and acetonitrile in the ratio 50:50 v/v for the $\mathrm{C} 18$ column tests and water:methanol in the ratio $50: 50 \mathrm{v} / \mathrm{v}$ for the biphenyl column tests. All solutions were freshly prepared and filtered using $0.22 \mu$ syringe filters, before use.

HPLC system used for method development and validation was Agilent Technologies 1260 Infinity module comprising of binary pump and degasser unit, combined with Agilent 1100 Series autosampler, autoinjector, column oven and VWD. The output signal was monitored and processed using Open Labs CDSChemstation Agilent software. A Phenomenex-Evo 100A C18 and a Restek Ultra Biphenyl column were used for the LC studies and method development.

\section{Detection, separation methods and validation:}


Satisfactory separations were achieved on the C18 column $(150 \times 4.6 \mathrm{~mm}, 2.6 \mu)$, by gradient elution using mobile phase comprising of water (A):acetonitrile (B) at a flow of $0.6 \mathrm{ml} \times \mathrm{min}^{-1}$. The gradient solvent program was set as (TT min/\% solution B) and $0 / 10,5 / 80$, $10 / 95,11.5 / 10,12.5 / 10$. The detection wavelength was $245 \mathrm{~nm}$. All mobile phases were filtered using $0.22 \mu \mathrm{m}$ filter papers (Kimix Chemical and Lab Supplies, Cape Town, South Africa). The column oven temperature was maintained at $27^{\circ}$ and the injection volume was $15 \mu \mathrm{l}$. A post runtime of 3 min was maintained between each injection.

The second separation was achieved on the biphenyl column $(150 \times 4.6 \mathrm{~mm}, 5 \mu)$, by same gradient elution solvents program as above, but with a mobile phase comprising of acetonitrile (A):methanol (B) instead of water, at a flow of $1 \mathrm{ml} \times \mathrm{min}^{-1}$ for a $15 \mathrm{~min}$ run. The detection wavelength was maintained at $245 \mathrm{~nm}$, with the same gradient $\mathrm{A}$ :B flow ratio as on the $\mathrm{C} 18$ column. The parameters for the HPLC runs were varied for both the columns (Table 1).

The method was validated for accuracy, linearity and precision. Limit of detection (LOD) and limit of quantification (LOQ) were determined based on signalto-noise ratio of 3 and 10, respectively, as per ICH guidelines ${ }^{[13]}$. The signal-to-noise ratio was determined by comparing measured signals from samples with known lowest concentrations of analyte with those of blank samples following three replicate injections and establishing the minimum concentration at which the analyte can be reliably detected or quantified. The method was validated for specificity using HLM assay, by incubating EFV as the substrate and NEO as the internal standard. Briefly, a standard $200 \mu \mathrm{l}$ incubation mixture containing liver microsomes $(0.5$ $\mathrm{mg} / \mathrm{ml}$ protein concentration), EFV $(100 \mu \mathrm{M})$ in $0.2 \mathrm{M}$ phosphate buffer $\mathrm{pH} 7.4$ at $37^{\circ}$ was incubated for 30, 45 and $60 \mathrm{~min}$, in duplicate. The reactions were initiated with NADPH (final concentration $1.3 \mathrm{mM}$ ) along with the co-factors $\mathrm{MgCl}_{2}$ (final concentration $3.3 \mathrm{mM}$ ), glucose-6-phosphate (final concentration $1.3 \mathrm{mM})$ and glucose-6-phosphate dehydrogenase $(1 \mathrm{U} / \mathrm{ml}$ ) and then terminated with $200 \mu \mathrm{l}$ of chilled acetonitrile spiked with the internal standard NEO $(10 \mu \mathrm{M})$. The samples were centrifuged at $10000 \mathrm{rpm}$ for $10 \mathrm{~min}$ and the supernatants were filtered in 0.22 $\mu \mathrm{m}$ centrifuge tube filters $\left(\right.$ Corning $^{\circledR} \operatorname{Costar}^{\circledR}$ Spin- $X^{\circledR}$ cellulose acetate membrane filters) and were subjected to HPLC analysis.

\section{TABLE 1: THE HPLC-VWD PARAMETERS FOR SEPARATION METHOD}

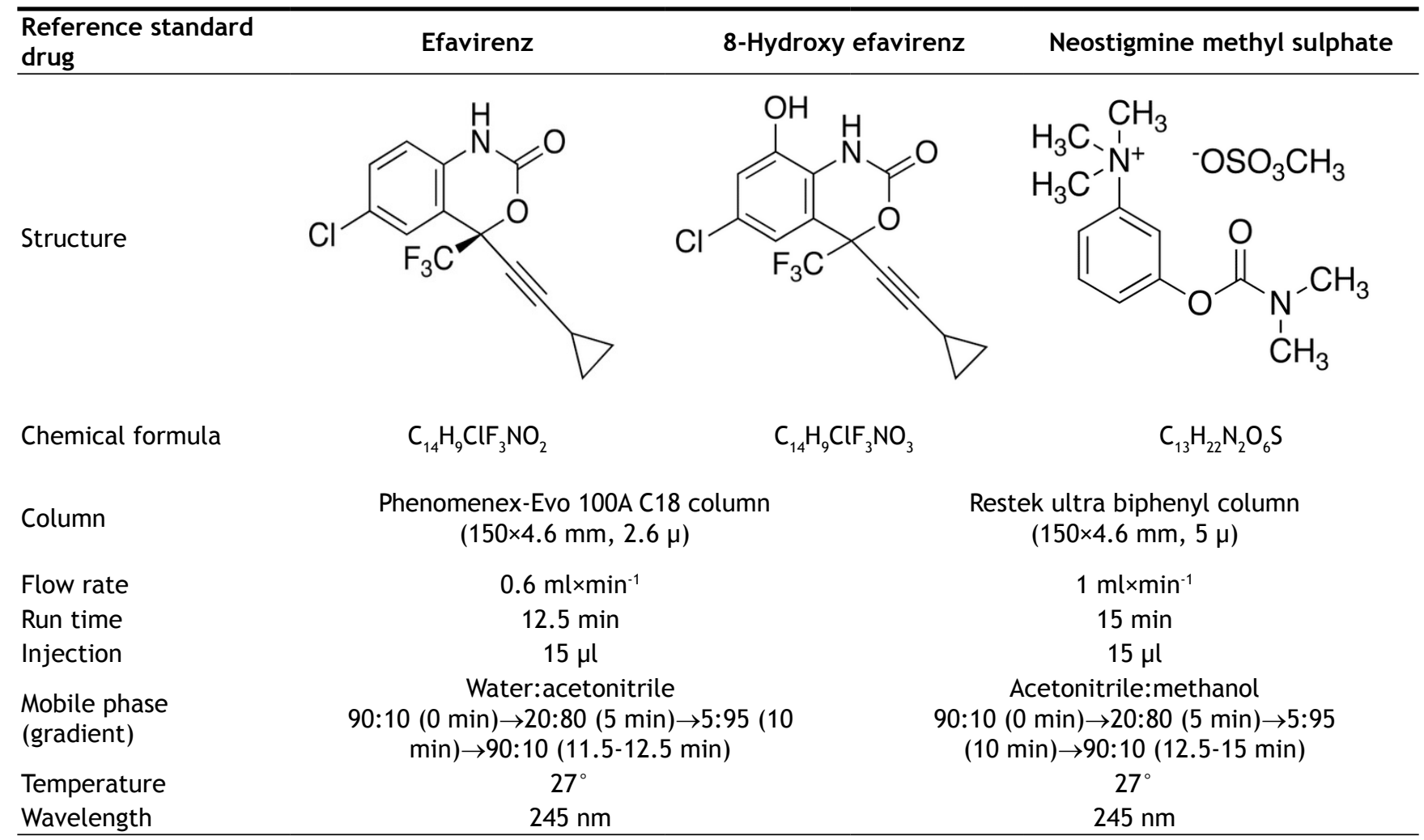

HPLC parameters for separation method for both C18 column and biphenyl column, structure of efavirenz, 8-hydroxy efavirenz and neostigmine 


\section{RESULTS AND DISCUSSION}

The literature search indicated that many HPLC methods were available for individual and a combination of two drugs. Based on the literature search, attempts were made to develop a simple method, which had less retention time and higher selectivity. Efforts were made to develop an isocratic method, but separation of the three drugs was not satisfactory; hence, gradient elution was selected ${ }^{[14]}$. Method optimization was done by different logical modifications such as change in column length (100, 150 and $250 \mathrm{~mm})$, inner diameter (2.6 and $4.6 \mathrm{~mm})$, particle size $(2.6,3.5$ and $5 \mu)$ and change in the gradient program. The results looked more stable in this method, suggesting that factors such as gradient methodology, mobile phases, column length, inner diameter and particle size were also decisive in better separation of these drugs and the conditions were found sufficient for the proposed method.

The separation of all the drugs was consistent and reproducible in both columns but the $\mathrm{C}-18$ column had a shorter run time (figs. 1 and 2) compared to the biphenyl column (fig. 3). The HPLC retention times were consistent at 8.75, 9.33 and $10.20 \mathrm{~min}$ for EFV, $\mathrm{E} 8 \mathrm{H}$ and NEO, respectively for the $\mathrm{C} 18$ column (Table 2). The C18 column was also consistent with lower concentrations of both EFV and E8H and provided faster separation compared to the biphenyl column. Hence the C18 column was used for further runs and method validation.

A linear response was obtained in the concentration 2.5-
$150 \mu \mathrm{M}$ for both EFV and E8H $\left(\mathrm{R}^{2}=0.99\right)$. NEO was maintained in same concentration in all HPLC runs, since the principle behind using this drug would be as an internal standard in the studies on drug metabolism using HLMs and the concentration of EFV used would be in the range of 50-100 $\mu \mathrm{M}$. LOD and LOQ were calculated at 32.33 and $96.97 \mu \mathrm{M}$ for EFV and 29.49 and $89.38 \mu \mathrm{M}$ for $\mathrm{E} 8 \mathrm{H}$, respectively. The results of the system suitability tests and the regression statistics (Table 3) assure the adequacy of the proposed HPLC method for routine analysis of EFV, E8H and NEO alone or in combination.

The percent relative standard deviation (RSD) values for intraday precision for both the standards as well as the in vitro incubation supernatants were less than $1 \%$. Intermediate precision was determined by carrying out the experiment by on a different system (HPLC Waters HPLC 2695 with 2996 PDA). Almost similar retention time was observed for all the three drugs.

Robust analytical methods are required in quality control laboratories for routine use, as well as in experiments involving drug interaction studies. After finalizing the mobile phase composition and column, statistically designed experiments were performed to screen robustness of an analytical method and to select the appropriate system suitability parameters. The use of the design of experiments (DOE) is essential in the robustness evaluation of method conditions and provides significant knowledge to establish optimal robust operating conditions as well as identify potential

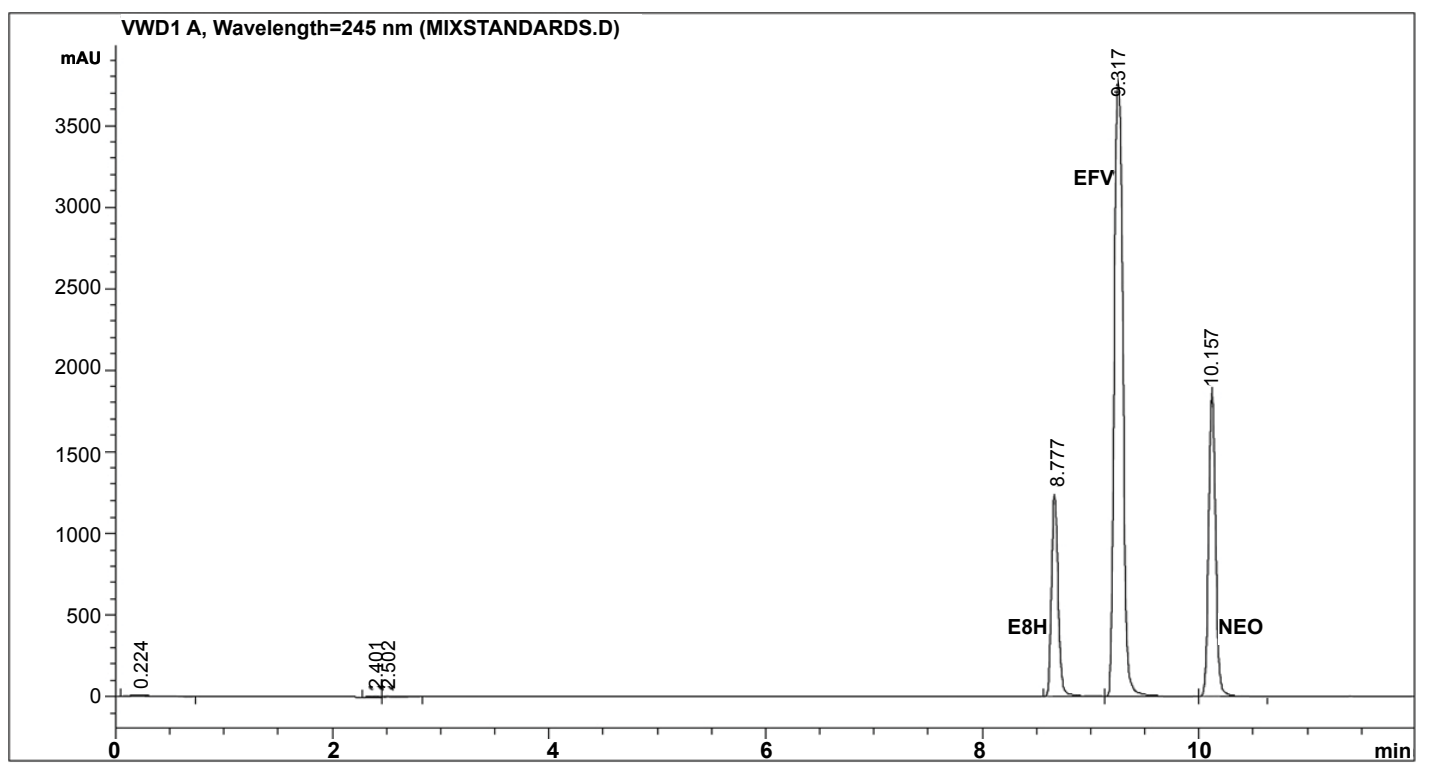

Fig. 1: HPLC chromatographic separation of E8H, EFV and NEO using the C18 column

Representative chromatogram of standards at $4 \mathrm{mM}$ concentration and their retention times on the C18 column, where efavirenz at $9.32 \mathrm{~min}, 8$-hydroxy efavirenz at $8.78 \mathrm{~min}$ and neostigmine at $10.16 \mathrm{~min}$ 


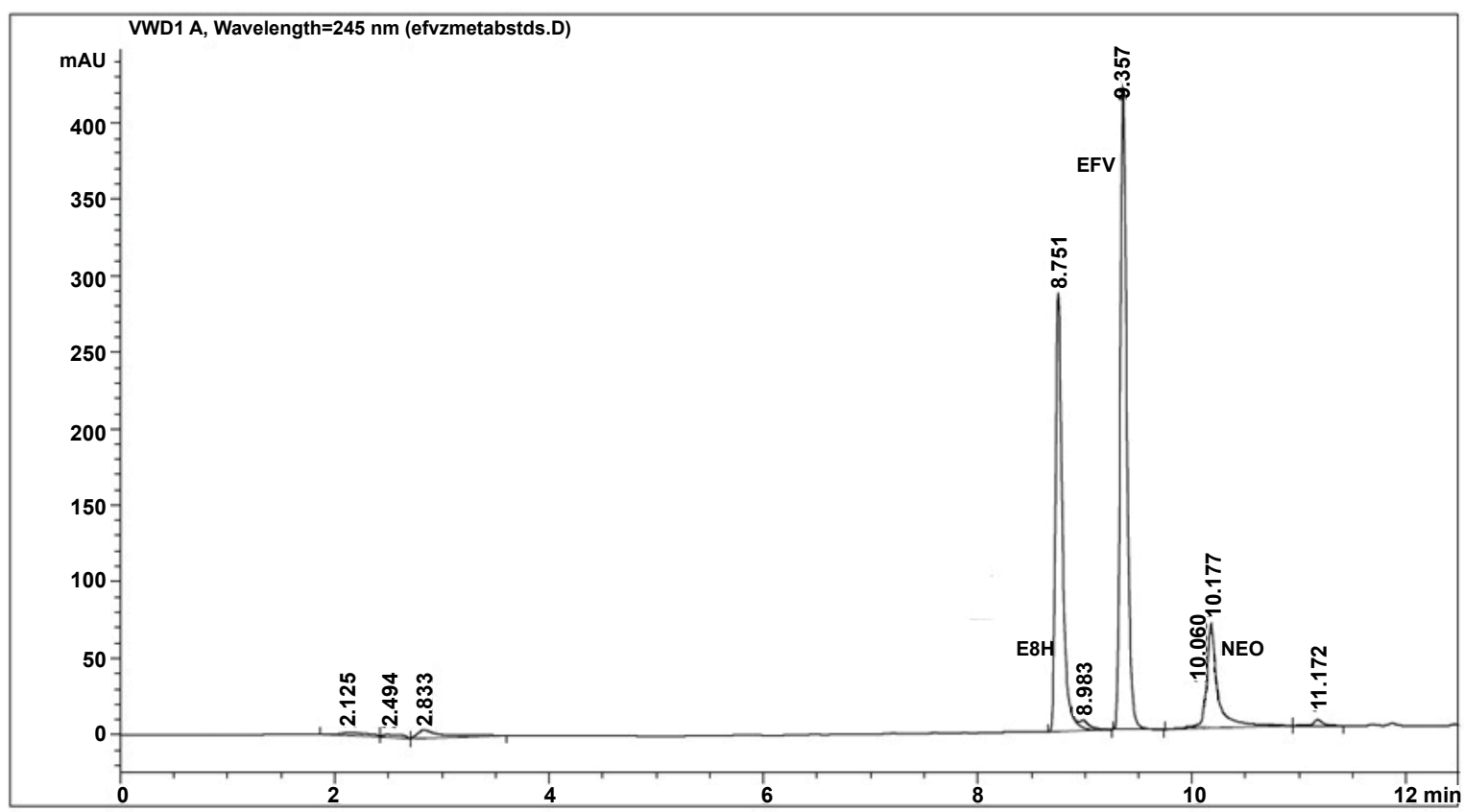

Fig. 2: HPLC separation of E8H, EFV and NEO using the C18 column

Representative chromatogram of standards at $200 \mu \mathrm{M}$ concentration and their retention times on the C18 column, where efavirenz at $9.36 \mathrm{~min}, 8$-hydroxy efavirenz at $8.75 \mathrm{~min}$ and neostigmine at $10.18 \mathrm{~min}$

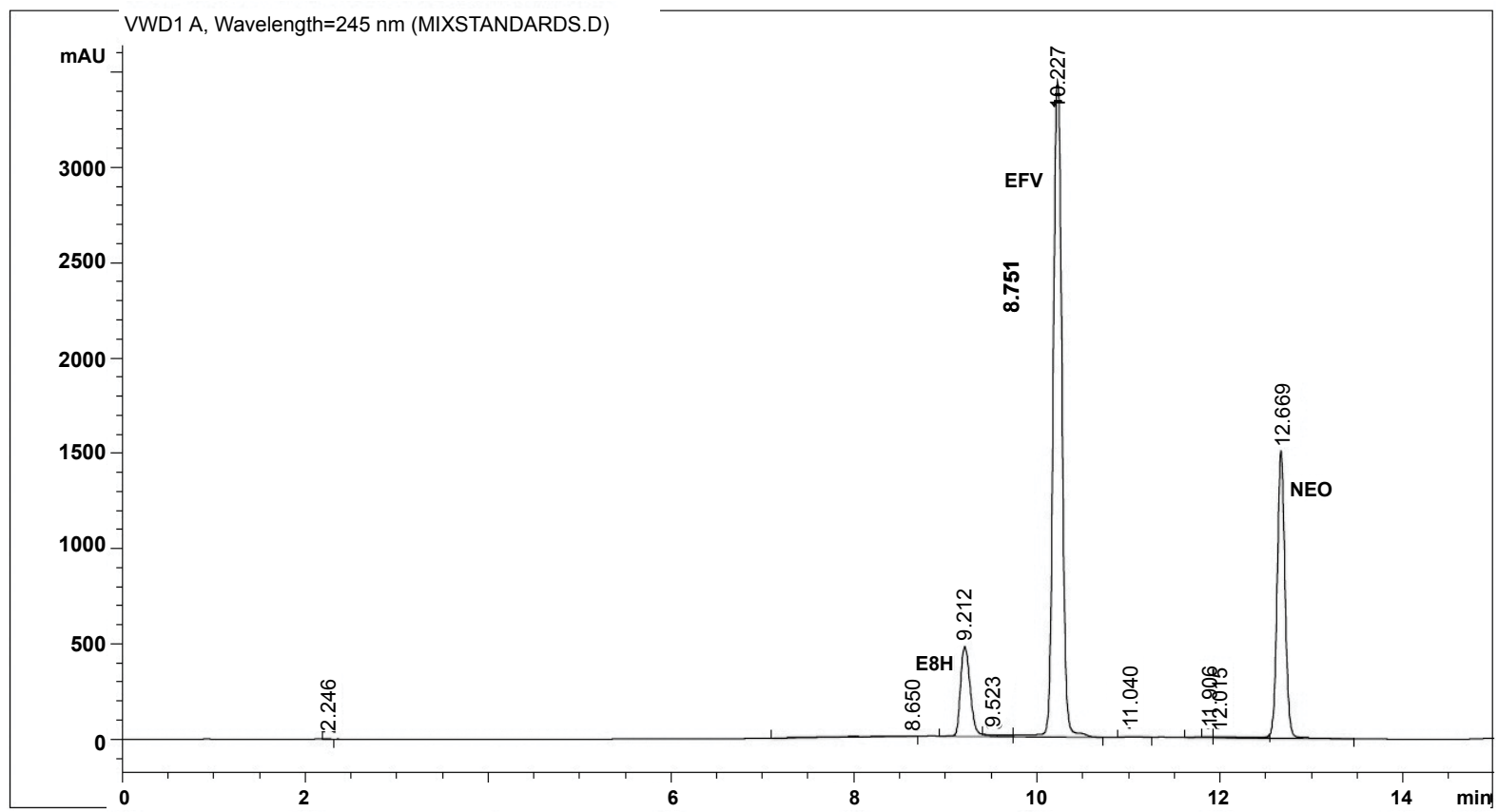

Fig. 3: HPLC separation of E8H, EFV and NEO using the biphenyl column

Representative chromatogram of standards at $4 \mathrm{mM}$ concentration and their retention times on the biphenyl column, where efavirenz at $\mathbf{1 0 . 2 3} \mathrm{min}, \mathbf{8}$-hydroxy efavirenz at $9.21 \mathrm{~min}$ and neostigmine at $\mathbf{1 2 . 6 7} \mathrm{min}$

failure modes. Various parameters such as flow rate of the mobile phase through the column, temperature of the column chamber and the detection wavelength were varied to validate the overall robustness of the method and the values for each factor showed consistent results (Table 4).

The applicability of the validated HPLC method for pharmacological drug metabolism studies was tested using in vitro human liver microsomal incubation assay. For all the three time slot incubations (30, 45 and $60 \mathrm{~min}$ ), the metabolite E8H was detected using this method at consistent retention times along with EFV, NEO and the peak area of NEO was relatively constant (fig. 4). Linearity was established for the 30-60 min 
incubation range based on the ratio of the metabolite to the internal standard, with $\mathrm{R}^{2}=0.97$ (Table 5).
The understanding gained from the method development and the robustness of experiments

TABLE 2: HPLC-VWD RUN RETENTION TIMES FOR BOTH COLUMNS

\begin{tabular}{|c|c|c|c|c|c|c|}
\hline \multirow{2}{*}{$\begin{array}{l}\text { Column } \\
\text { Drug }\end{array}$} & \multicolumn{3}{|c|}{$\begin{array}{c}\text { Phenomenex-Evo C18 100A column } \\
(150 \times 4.6 \mathrm{~mm}, 2.6 \mu)\end{array}$} & \multicolumn{3}{|c|}{$\begin{array}{l}\text { Restek ultra biphenyl column } \\
(150 \times 4.6 \mathrm{~mm}, 5 \mu)\end{array}$} \\
\hline & EFV & $\mathrm{E} 8 \mathrm{H}$ & NEO & EFV & $\mathrm{E} 8 \mathrm{H}$ & NEO \\
\hline Retention time (min) & 8.75 & 9.33 & 10.20 & 9.20 & 10.22 & 12.66 \\
\hline Overall run time (min) & \multicolumn{3}{|c|}{12.5} & \multicolumn{3}{|c|}{15} \\
\hline
\end{tabular}

HPLC overall run-time and retention times for efavirenz, 8-hydroxy efavirenz and neostigmine on both C18 and biphenyl columns

\section{TABLE 3: REGRESSION STATISTICS FOR STANDARDS ON C18 COLUMN}

\begin{tabular}{lcc}
\hline Statistical criteria & EFV & E8H \\
\hline Linearity range $(\mu M)$ & $2.5-150$ & $2.5-150$ \\
Linear correlation coefficient $\left(\mathrm{R}^{2}\right)$ & 0.915713 & 0.9275 \\
Polynomial correlation coefficient $\left(\mathrm{R}^{2}\right)$ & 0.9993 & 0.9991 \\
Standard error & 116.6889 & 54.2463246 \\
Intercept coefficient & -88.722 & -44.78345 \\
LOD $(\mu M)$ & 32.33 & 29.49 \\
LOQ $(\mu M)$ & 96.97 & 89.38 \\
\%RSD & 0.918 & 0.982 \\
\hline
\end{tabular}

Limit of detection (LOD) and limit of quantification (LOQ) and regression data for both efavirenz and 8-hydroxy efavirenz chromatograms

TABLE 4: ROBUSTNESS EVALUATION PARAMETERS FOR C18 COLUMN RUNS

\begin{tabular}{lcccc}
\hline \multirow{2}{*}{ Parameter } & \multirow{2}{*}{ Variation } & \multicolumn{3}{c}{ Retention time (min) } \\
\cline { 2 - 4 } Flow rate & $0.6 \mathrm{~m} \times \mathrm{min}^{-1}$ & 8.75 & E8H & NEO \\
& $0.7 \mathrm{ml} \times \mathrm{min}^{-1}$ & 8.62 & 9.33 & 10.20 \\
\multirow{3}{*}{ Temperature } & $0.8 \mathrm{ml}^{*} \mathrm{~min}^{-1}$ & 8.53 & 9.21 & 10.10 \\
& $27^{\circ}$ & 8.75 & 9.12 & 10.01 \\
\multirow{3}{*}{ Wavelength } & $30^{\circ}$ & 8.69 & 9.33 & 10.20 \\
& $32^{\circ}$ & 8.79 & 9.39 & 10.28 \\
& $245 \mathrm{~nm}$ & 8.75 & 9.21 & 10.19 \\
\multicolumn{2}{c}{ \%RSD retention time } & 8.70 & 9.33 & 10.20 \\
& $247 \mathrm{~nm}$ & 8.69 & 9.28 & 10.29 \\
\hline
\end{tabular}

Retention times for efavirenz, 8-hydroxy efavirenz and neostigmine, for various parameter alteration-runs on the C18 column

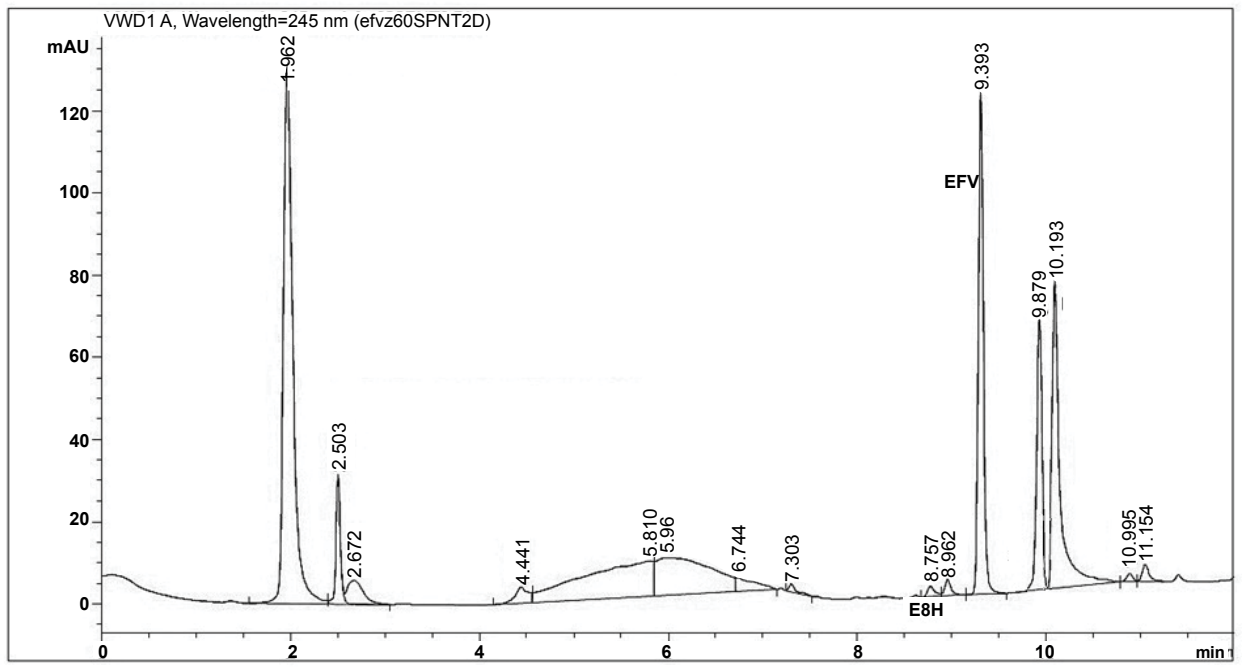

Fig. 4: HPLC separation of E8H, EFV and NEO using the C-18 column, for the in vitro HLM-EFV incubation sample Representative chromatogram of supernatant from HLM assay and the retention times of the resultant on the C18 column, where efavirenz at $9.39 \mathrm{~min}, 8$-hydroxy efavirenz at $8.76 \mathrm{~min}$ and neostigmine at $10.19 \mathrm{~min}$ 


\begin{tabular}{lc}
\hline Statistical criteria & E8H:NEO Ratio \\
\hline Incubation time-linearity range $(\mathrm{min})$ & 30,45 and 60 \\
Linear correlation coefficient $\left(\mathrm{R}^{2}\right)$ & 0.968 \\
Standard error & 0.001 \\
Intercept coefficient & 0.0082 \\
\%RSD retention times & $0.23 \quad 0.45 \quad 0.16$ \\
& \\
\end{tabular}

Regression data for efavirenz, 8-hydroxy efavirenz and neostigmine in the in vitro assay analysis-chromatograms for the 30,45 and 60 min incubations

enabled a choice of column for optimised resolution and provided assurance of separation of all the drugs, within a shorter run time. The chromatography yielded well-resolved peaks for EFV, E8H and NEO, with good accuracy, precision and recovery and the same was validated for specificity on the HLM incubations, where the results proved consistent. This HPLC-VWD method is suitable for therapeutic drug monitoring in patients with HIV/AIDS alone or co-infected with myasthenia gravis in a less resourced setting, studies involving in vitro metabolism of EFV and its major metabolites, as well as warranted in pharmacokinetic studies involving drug-drug and herb-drug interactions. Very few gradient methods were available on the HPLC-VWD for the separation of EFV and its major metabolites from in vitro assays involving HLM; the novelty of this method being the only reversegradient method using normal solvents (no buffers or formic acid) such as acetonitrile and water/methanol for a perfect separation of EFV and $\mathrm{E} 8 \mathrm{H}$, along with NEO as a marker internal standard on a C18 EVO $2.6 \mu$ column as well as a biphenyl $5 \mu$ column. The success of this method in handling a complex matrix as the in vitro assay sample, by separating the drug/ metabolite phase and the internal standard phase from the other components of the assay without using SPE methodologies, makes it simple, efficient and less time consuming.

\section{Acknowledgments:}

The authors are grateful to the National Research Foundation Agency of South Africa (NRF IKS Grant No: 82641) for the funding. The authors also wish to thank the representatives of LECO Africa, Cape Town in association with Restek Corporation USA, for all their support in the supply of the column and the research study.

\section{Financial assistance:}

This research is funded by the National Research
Foundation agency of South Africa, Indigenous Knowledge Systems, Grant No: 82641.

\section{Conflict of interests:}

None declared.

\section{REFERENCES}

1. De Clercq E. Antiviral drugs: current state of the art. J Clin Virol 2001;22:73-89.

2. Mutlib AE, Chen H, Nemeth GA, Markwalder JA, Seitz SP, Gan LS, et al. Identification and characterization of efavirenz metabolites by liquid chromatography/mass spectrometry and high field NMR: species differences in the metabolism of efavirenz. Drug Metab Dispos 1999;27:1319-33.

3. Ward BA, Gorski JC, Jones DR, Hall SD, Flockhart DA, Desta $\mathrm{Z}$. The cytochrome $\mathrm{P}_{450}$ 2B6 (CYP2B6) is the main catalyst of efavirenz primary and secondary metabolism: implication for HIV/AIDS therapy and utility of efavirenz as a substrate marker of CYP2B6 catalytic activity. J Pharmacol Exp Ther 2003;306:287-300.

4. Mogatle S, Kanfer I. Rapid method for the quantitative determination of efavirenz in human plasma. J Pharma Biomed Anal 2009;49:1308-12.

5. Ramachandran G, Kumar AK, Swaminathan S, Venkatesan P, Kumaraswami V, Greenblatt DJ. Simple and rapid liquid chromatography method for determination of efavirenz in plasma. J Chromatogr B Analyt Technol Biomed Life Sci 2006;835:131-5.

6. Saras-Nacenta M, Lopez-Pua Y, Lopez-Cortes LF, Mallolas J, Gatell JM, Carne X. Determination of efavirenz in human plasma by high performance liquid chromatography with ultraviolet detection. J Chromatogr B Biomed Sci Appl 2001;763:53-9.

7. Ogburn ET, Jones DR, Masters AR, Xu C, Guo Y, Desta Z. Efavirenz primary and secondary metabolism in vitro and in vivo: identification of novel metabolic pathways and cytochrome $\mathrm{P}_{450} 2 \mathrm{~A} 6$ as the principal catalyst of efavirenz 7-hydroxylation. Drug Metab Disp 2010;38:1218-29.

8. Önal A. Analysis of antiretroviral drugs in biological matrices for therapeutic drug monitoring. J Food Drug Anal 2006;14:99-119.

9. Dimeski G. Interference testing. Clin Biochem Rev 2008;29:43-8.

10. https://www.fda.gov/downloads/Drugs/.../Guidances/ ucm079266.pdf.

11. Haynes BF, Hale LP, Weinhold KJ, Patel DD, Liao HX, Bressler 
$\mathrm{PB}$, et al. Analysis of the adult thymus in reconstitution of $\mathrm{T}$ lymphocytes in HIV-1 infection. J Clin Invest 1999;103:453-60.

12. Gorthi SP, Shankar S, Johri S, Mishra A, Chaudhary NR. HIV infection with myasthenia gravis. J Assoc Physicians India 2005;53:995-6.
13. https://www.ich.org/fileadmin/Public_Web_Site/ICH Products/Guidelines/Quality/Q2 R1/Step4/Q2 R1 Guideline.pdf.

14. Varghese A, Pandita N, Gaud RS. In vitro and in vivo Evaluation of CYP1A Interaction Potential of Terminalia arjuna Bark. Indian J Pharm Sci 2014;76:138-47. 